\title{
Polymorphisms of the Chicken Mx Gene Promoter and Association with Chicken Embryos' Susceptibility to Virulent Newcastle Disease Virus Challenge
}

\author{
Fulgence N. Mpenda $\left(\mathbb{D},{ }^{1}\right.$ Christian T. Keambou, ${ }^{2}$ Martina Kyallo, ${ }^{2}$ Roger Pelle, ${ }^{2}$ \\ Sylvester L. Lyantagaye, ${ }^{3}$ and Joram Buza ${ }^{1}$ \\ ${ }^{1}$ School of Life Sciences and Bioengineering, Nelson Mandela African Institution of Science and Technology, P.O. Box 447, \\ Tengeru, Arusha, Tanzania \\ ${ }^{2}$ Biosciences Eastern and Central Africa, International Livestock Research Institute, Nairobi, Kenya \\ ${ }^{3}$ Department of Molecular Biology and Biotechnology College of Natural and Applied Sciences, University of Dar es Salaam, \\ Dar es Salaam, Tanzania \\ Correspondence should be addressed to Fulgence N. Mpenda; mpenda83@gmail.com
}

Received 6 July 2019; Revised 26 August 2019; Accepted 31 August 2019; Published 3 October 2019

Academic Editor: Paolo Muratori

Copyright ( $\odot 2019$ Fulgence N. Mpenda et al. This is an open access article distributed under the Creative Commons Attribution License, which permits unrestricted use, distribution, and reproduction in any medium, provided the original work is properly cited.

\begin{abstract}
Newcastle disease is a devastating viral disease of chicken in low- and middle-income countries where the backyard production system is predominant. Marker-assisted selection of chickens that are resistant to Newcastle disease virus (NDV) is the promising strategy that needs to be explored. The aim of the present study was to investigate polymorphisms of the promoter region of the chicken Mx gene and association with Kuroiler, Sasso, and local Tanzanian chicken embryos' survival variability to virulent NDV infection. Chicken embryos were initially challenged with a minimum lethal dose of virulent NDV suspension and then were followed over time to gather information on their survival variability. Using the survival data, high and less susceptible cohorts were established, and a total of 88 DNA samples from high and less susceptible groups were genotypes by sequencing. Five singlenucleotide polymorphisms (SNPs), which were previously reported, were detected. Interestingly, for the first time, the findings demonstrated the association of the promoter region of chicken myxovirus-resistance $(\mathrm{Mx})$ gene polymorphisms with chicken embryos' susceptibility to the virulent NDV challenge. At the genotypic level, the SNP4 $G>A$ mutation that was located within the IFN-stimulating response element was associated (LR: $6.97, P=0.03$ ) with chicken embryos' susceptibility to the virulent NDV challenge. An allele $G$ frequency was higher in the less susceptible cohort, whereas an allele $A$ frequency was higher in the high susceptible cohort. At the haplotype level, the haplotype group ACGC was associated (OR: 9.8, 95\% CI: 1.06-79.43, $P=0.042$ ) with the same trait and had a resistant effect. In conclusion, the results have demonstrated the association of chicken Mx gene promoter polymorphisms and chicken embryos' survival variability to the virulent NDV challenge, and the information is useful for breeding programs designed to develop chicken genotypes that are resistant to Newcastle disease virus.
\end{abstract}

\section{Introduction}

The myxovirus-resistance $(\mathrm{Mx})$ genes are found in a wide range of living organisms including chicken [1]. Mx proteins are interferon- (IFN-) induced GTPase enzymes with antiviral functions, which particularly play a significant role in inhibition of negative-stranded RNA viruses $[1,2]$. Binding of type I or III IFNs to receptors triggers the expression of
IFN-stimulated genes, thereby inducing an antiviral state within a cell. Therefore, the expression of Mx genes largely depends on the activation of type I or III IFNs $[1,3]$.

The genomic size of the chicken Mx gene is about $21 \mathrm{kbps}$ with the coding sequences of about 2118 bps encoding the Mx protein comprising 705 amino acids $[4,5]$. Like other IFN-response genes, the chicken $\mathrm{Mx}$ gene contains a sequence element in its promoters that serve as inducible 
enhancers. The chicken $\mathrm{Mx}$ gene promoter contains a motif $5^{\prime}$-AGGTTTCTTTCCT- 3 ' or its reverse complement $[6,7]$, which is an integral part of the IFN-stimulated response element (ISRE). It has been documented that the ISRE motif has a crucial role to play in IFN inducibility of the chicken Mx gene [6].

Multiple allelic variants of the chicken $\mathrm{Mx}$ gene have been reported in different populations of chicken throughout the world $[4,8,9]$. For example, Li et al. [4] reported a total of 24 single-nucleotide polymorphisms (SNPs) after comparison of four chicken sequences. The highest nucleotide diversity ( $\pi$ value: 0.01003 ) was in the chicken Mx gene promoter where a total of six SNPs were found [4]. A similar finding was observed when nine elite egg-layer-type lines were sequenced where a total of 6 SNPs out of 36 SNPs that were reported were found in the chicken Mx gene promoter [9].

Most of the available reports have demonstrated the role of the chicken Mx gene G2032A (S631N) in antiviral activities, particularly against influenza virus [10-12]; however, a considerable number of reports failed to demonstrate the same association $[8,13]$. These conflicting reports may suggest that the antiviral activity of the chicken $\mathrm{Mx}$ gene involves several allelic variants, and more remain to be elucidated. Therefore, the present study was aimed to investigate the polymorphisms of the chicken Mx gene promoter and association with Kuroiler, Sasso, and local Tanzanian chicken embryos' survival variability to the virulent NDV challenge.

\section{Materials and Methods}

2.1. Source of the Virulent Newcastle Disease Virus (NDV) Isolate. A virulent NDV field isolate from live birds was kindly provided by Sokoine University of Agriculture. Characterization was done as previously described [14-17] to confirm virulence of the strain before the experiment was conducted. The virus was then titrated to a working titre of a minimum lethal dose (MLD) of $10^{3} / 0.1 \mathrm{~mL}$ of virus suspension, and the viral suspension was stored at $-80^{\circ} \mathrm{C}$ until use.

2.2. Embryonated Chicken Eggs' Challenge. The experiment involved three chicken types, which had the same history of NDV immunization: Tanzanian local chicken, Kuroiler [18], and Sasso [19]. Chicken embryos'variability in susceptibility to virulent NDV infection was evaluated by detection of chicken embryo survival time following infection with the MLD of the viral suspension. Therefore, sixteen-day-old embryonated eggs were inoculated with $0.1 \mathrm{ml}\left(10^{3} \mathrm{MLD} /\right.$ $0.1 \mathrm{~mL}$ ) of virus suspension directly deposited into the allantoic fluid by using a $1 \mathrm{ml}$ sterile syringe. Eggs were sealed with adhesive glue and incubated at $37.9^{\circ} \mathrm{C}$ for a total of 120 hours after infection (pi). During this time, except for first 24 hours after infection, embryonated eggs were candled at the time interval of 6 hours to detect dead embryos. Dead embryonated eggs were chilled at $4^{\circ} \mathrm{C}$ for four hours before tissue harvest. In the experiment, a total of 355 (87 Sasso, 129
Kuroiler, and 139 local chicken) embryonated eggs were challenged in three experimental replicates.

\subsection{Chicken Embryo Tissue Harvest and DNA Extraction.} In a biological safety cabinet, dead embryonated eggs were decontaminated by using $70 \%$ ethanol. Then, leg muscles and comb were harvested and stored in separate tubes, which were then stored at $-20^{\circ} \mathrm{C}$ for genomic DNA extraction. The selective genotyping approach was employed as it was previously demonstrated $[20,21]$. Thus, using chicken embryos' survival data, genomic DNA was extracted from high (15\%) and less (15\%) susceptible groups. Genomic DNA was extracted from comb or leg tissues using the Quick-DNA Tissue/Insect Kit (Zymo Research) in accordance with the manufacturer's instructions. The quality and quantity of genomic DNA were assessed by running on $1 \%$ (w/v) agarose gel containing ethidium bromide in $0.5 \% \mathrm{TBE}$ buffer for an hour.

2.4. PCR Amplification. The polymerase chain reaction (PCR) was conducted to amplify a DNA fragment of about $284 \mathrm{bp}$ region of the $5^{\prime}$ untranslated region and partial promoter of the chicken Mx gene [4]. The selected primers (forward primer: 5'-ACCTGTGCCATCTGCCCTCTGA-3' and reverse primer: 5'-CACAGCAAGGAGAAACAATTAACTACAT- $3^{\prime}$ ) and PCR conditions were as previously described [22]. The amplification was conducted in a reaction volume of $25 \mu \mathrm{L}$ containing $0.2 \mu \mathrm{M}$ of each primer and $12.5 \mu \mathrm{L}$ of Taq 2X PCR MasterMix (New England Biolabs (NEB)). The PCR conditions were as follows: initial denaturation at $94^{\circ} \mathrm{C}$ for 5 minutes, followed by 40 cycles of denaturation at $94^{\circ} \mathrm{C}$ for 1 minute, annealing at $58.5^{\circ} \mathrm{C}$ for 30 seconds, extension at $72^{\circ} \mathrm{C}$ for 1 minute, and final extension at $72^{\circ} \mathrm{C}$ for 5 minutes. The PCR ran on the QuantStudio 6 Flex Real-Time (RT) PCR Thermal Cycler (Applied Biosystems). The quality of PCR products was evaluated by running on $1 \%(\mathrm{w} / \mathrm{v})$ agarose gel containing ethidium bromide in $0.5 \mathrm{X}$ TBE buffer at $100 \mathrm{~V}$ for an hour.

2.5. Sequencing and Bioinformatics Analysis. Raw sequences were trimmed using CLC Genomics Workbench v3.0.8 and consensus sequences generated. A total of 88 sequences ( 24 Kuroiler, 32 local chicken, and 32 Sasso) were further analyzed. Multiple-sequence alignment was done using the MUSCLE algorithm in MEGA v6 to identify polymorphic sites. SNP calling was done using CLC Genomics Workbench v3.0.8.

2.6. Statistical Analysis. Population genetics parameters like the Hardy-Weinberg equilibrium, pairwise linkage disequilibrium, and association analysis were performed by using SNPStats, a web tool for SNP analysis [23]. Other statistic tests like Pearson's chi-squared test of independence of genotype frequencies and likelihood ratio tests were conducted by using the $\mathrm{R}$ software (version 3.3.3; The $\mathrm{R}$ Foundation for Statistical Computing). 


\section{Results}

3.1. Chicken Embryos' Survival Time after Challenge with Virulent NDV. The survival time probability of developing chicken embryos upon challenge with virulent NDV is presented in Figure 1. Overall, Sasso chicken embryos had the highest survival time probability as compared to Kuroiler and local Tanzanian chicken embryos (Figure 1).

\subsection{Sequence Variations in the Amplified Promoter Region of} the Chicken Mx Gene. A total of five single-nucleotide polymorphisms (SNPs) were observed in the present study (Table 1). All SNPs were previously reported when the same (284 bp) promoter region of the chicken Mx gene was sequenced [22]. Generally, the observed and expected heterozygosity for all SNPs was at the same levels (Table 1). Furthermore, all the SNPs had no interaction between the response variable (susceptibility) and the covariate (breed), with the exception of SNP3. Additionally, SNP3 was not associated with chicken embryos' variability in susceptibility to virulent NDV infection, and therefore, SNP3 was removed from further analysis to allow breed merging.

3.3. The Hardy-Weinberg Equilibrium. The 5 SNPs were tested for agreement with the Hardy-Weinberg equilibrium (HWE). All SNPs were in consistence with the HardyWeinberg principle $(P>0.05)$ (Table 2). Also, the minor allele frequencies in high $(\mathrm{H})$ and less $(\mathrm{L})$ susceptible cohorts were greater than 0.05 (Table 2).

3.4. SNP Allele and Genotype Frequency. The allele and genotype frequencies of 4 SNPs (SNP1, SNP2, SNP4, and SNP5) are presented in Tables 2 and 3. At the allelic level, the results indicated that there was not association between SNPs and chicken embryos' susceptibility to the virulent NDV challenge. However, at the genotypic level, SNP4 (LR $=6.97, P<0.05)$ was significantly associated with chicken embryos' susceptibility to virulent NDV infection (Table 3).

3.5. Linkage Disequilibrium and Haplotype Frequency. Results of linkage disequilibrium (LD) of 5 SNPs are shown in Table 4. All the SNPs were in LD $(P<0.05)$ with the exception of SNP3 $(P>0.05)$, which was in equilibrium with other SNPs (Table 4).

Haplotype analysis of four SNPs that were in LD generated 4 haplotypes (Table 5). The haplotype group " $A C G T$ " had highest haplotype frequency (0.74), and the lowest haplotype frequency (0.01) was observed in the haplotype group "AGGT." The haplotype group " $A C G C$," which had a frequency of 0.06 , was associated $(P<0.05)$ with chicken embryos' susceptibility to virulent NDV infection (Table 5).

\section{Discussion}

In the present study, a combination of candidate gene and selective genotyping approaches were employed to
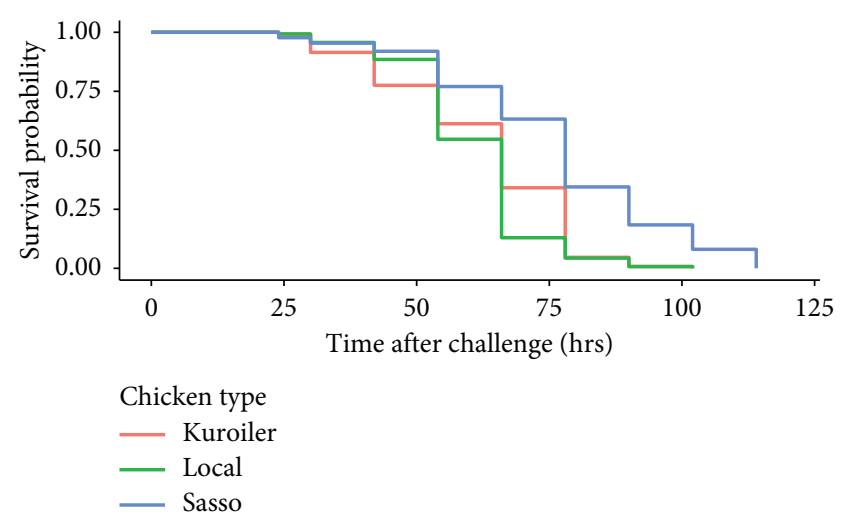

FIGURE 1: Kaplan-Meier survival curves of chicken embryos following the challenge with virulent Newcastle disease virus observed in the present study.

TABLE 1: Single-nucleotide polymorphic sites observed in the study.

\begin{tabular}{lcccc}
\hline Markers & Position & $H_{\mathrm{O}}$ & $H_{\mathrm{E}}$ & Allele change \\
\hline SNP1 & $91^{\text {st }}$ & 0.34 & 0.28 & $A>T$ \\
SNP2 & $107^{\text {th }}$ & 0.34 & 0.32 & $C>G$ \\
SNP3 & $113^{\text {th }}$ & 0.34 & 0.38 & $C>T$ \\
SNP4 & $194^{\text {th }}$ & 0.28 & 0.30 & $G>A$ \\
SNP5 & $231^{\text {st }}$ & 0.31 & 0.37 & $T>C$ \\
\hline
\end{tabular}

$H_{\mathrm{O}}$ : observed heterozygosity; $H_{\mathrm{E}}$ : expected heterozygosity. Position is the polymorphic site corresponding to 1 to 284 positions along the amplified product length from $5^{\prime}$ to $3^{\prime}$.

investigate polymorphisms of the promoter region of the chicken Mx gene and association with chicken embryos' susceptibility to virulent NDV infection. Chicken embryos were initially challenged with the MLD of virulent NDV suspension, and information was continuously gathered on their survival variability pi. Using the survival data, high and less susceptible cohorts were established for the promoter region of chicken $\mathrm{Mx}$ gene genotyping by sequencing. We were able to detect 5 SNPs, which had been reported previously by Mishra et al. [22].

Interestingly, for the first time, the findings demonstrated the association of the promoter region of chicken $\mathrm{Mx}$ gene polymorphisms with chicken embryos' susceptibility to virulent NDV infection. At the genotypic level, SNP4 was associated $(P<0.05)$ with the phenotype, whereas at the haplotype level, the haplotype group $A C G C$ was associated $(P<0.05)$ with the phenotype. The SNP4 $G>A$ mutation was detected at the $194^{\text {th }}$ position (Table 1 ) corresponding to 1 to 284 positions along the amplified product length polymorphisms for $5^{\prime}$ to $3^{\prime}$. SNP4 was within the AGTTTCGTTTCT motif of the ISRE, and the SNP was previously reported in the same position [4]. The ISRE plays a crucial role in IFN inducibility of the chicken Mx gene [6]. The results of the present study demonstrated the association (LR: 6.97, $P=0.03$ ) of the genotype $G G$ of SNP4 with less susceptibility of chicken embryos to virulent NDV infection. The genotype $G G$ frequency of 0.7 in the less susceptible group was higher as compared to the genotype $G G$ frequency of 0.63 in the high susceptible group. Likewise, although it was not statistically significant, allele $G$ had a higher 
TABLE 2: Allele frequency of polymorphic sites of the promoter region of the chicken Mx gene and association with chicken embryos' susceptibility to virulent NDV infection.

\begin{tabular}{|c|c|c|c|c|c|c|c|}
\hline \multirow{2}{*}{ Markers } & \multirow{2}{*}{ Allele } & \multicolumn{2}{|c|}{ Susceptibility } & \multirow{2}{*}{$P$ value $\left(\chi^{2}\right)$} & \multirow{2}{*}{$P$ value (LR) } & \multirow{2}{*}{$P$ value (HWE) } & \multirow{2}{*}{ MAF } \\
\hline & & $\mathrm{H}$ & $\mathrm{L}$ & & & & \\
\hline \multirow{2}{*}{ SNP1 } & $A$ & $59(0.78)$ & $85(0.85)$ & 0.02 & 0.01 & \multirow{2}{*}{1} & \multirow{2}{*}{0.16} \\
\hline & $T$ & $17(0.22)$ & $15(0.15)$ & 0.89 & 0.09 & & \\
\hline \multirow{2}{*}{ SNP2 } & C & $58(0.76)$ & $84(0.84)$ & 1.64 & 1.62 & \multirow{2}{*}{1} & \multirow{2}{*}{0.20} \\
\hline & $G$ & $18(0.24)$ & $16(0.16)$ & 0.20 & 0.2 & & \\
\hline \multirow{2}{*}{ SNP4 } & $G$ & $58(0.76)$ & $85(0.85)$ & 2.14 & 2.12 & \multirow{2}{*}{0.49} & \multirow{2}{*}{0.19} \\
\hline & $A$ & $18(0.24)$ & $15(0.15)$ & 0.14 & 0.14 & & \\
\hline \multirow{2}{*}{ SNP5 } & $T$ & $58(0.76)$ & $75(0.75)$ & 0.04 & 0.04 & \multirow{2}{*}{0.14} & \multirow{2}{*}{0.24} \\
\hline & C & $18(0.24)$ & $25(0.25)$ & 0.84 & 0.84 & & \\
\hline
\end{tabular}

$\chi^{2}$, Pearson's chi-squared test of independence; LR, Likelihood ratio; HWE, Hardy-Weinberg equilibrium test; H, high susceptible chicken embryo group; L, less susceptible chicken embryo group; MAF, minor allele frequency.

TABLE 3: Genotype frequency of polymorphic sites of the chicken Mx gene promoter and association with chicken embryos' susceptibility to virulent NDV infection.

\begin{tabular}{|c|c|c|c|c|c|}
\hline \multirow{2}{*}{ Markers } & \multirow{2}{*}{ Genotype } & \multicolumn{2}{|c|}{ Susceptibility } & \multirow{2}{*}{$P$ value $\left(\chi^{2}\right)$} & \multirow{2}{*}{$P$ value (LR) } \\
\hline & & $\mathrm{H}$ & $\mathrm{L}$ & & \\
\hline \multirow{3}{*}{ SNP1 } & $A A$ & $24(0.63)$ & $35(0.7)$ & 4.11 & 5.20 \\
\hline & $A T$ & $11(0.29)$ & $15(0.3)$ & 0.13 & 0.07 \\
\hline & $T T$ & $3(0.08)$ & - & & \\
\hline \multirow{3}{*}{ SNP2 } & $C C$ & $23(0.61)$ & $34(0.68)$ & 4.13 & 5.23 \\
\hline & $C G$ & $12(0.32)$ & $16(0.32)$ & 0.13 & 0.07 \\
\hline & $G G$ & $3(0.08)$ & - & & \\
\hline \multirow{3}{*}{ SNP4 } & $A A$ & $4(0.11)$ & - & 5.52 & 6.97 \\
\hline & $A G$ & $10(0.26)$ & $15(0.3)$ & 0.04 & 0.03 \\
\hline & $G G$ & $24(0.63)$ & $35(0.7)$ & & \\
\hline \multirow{3}{*}{ SNP5 } & $C C$ & $4(0.11)$ & $4(0.08)$ & 0.67 & 0.67 \\
\hline & $C T$ & $10(0.26)$ & $17(0.34)$ & 0.72 & 0.72 \\
\hline & $T T$ & $24(0.63)$ & $29(0.58)$ & & \\
\hline
\end{tabular}

$\chi^{2}$, Pearson's chi-squared test of independence; LR, likelihood ratio.

TABLE 4: The linkage disequilibrium $r$ statistic for five SNPs reported in the present study.

\begin{tabular}{lccccc}
\hline & SNP1 & SNP2 & SNP3 & SNP4 & SNP5 \\
\hline SNP1 & - & 0.96 & -0.28 & 0.98 & 0.83 \\
SNP2 & - & - & -0.29 & 0.94 & 0.79 \\
SNP3 & - & - & - & -0.28 & 0.07 \\
SNP4 & - & - & - & - & 0.81 \\
SNP5 & - & - & - & - & - \\
\hline
\end{tabular}

frequency (0.85) in the less susceptible group as compared with the allele $G$ frequency (0.76) in the high susceptible group. Also, allele $A$ had high frequency (0.24) in the high susceptible group as compared with the allele $A$ frequency (0.15) in the less susceptible group. It can be said that the mutation of $G>A$ is associated with susceptibility of chicken embryos to virulent NDV infection, whereas allele $G$ is associated with resistance of the same trait. The mechanisms underlying this observation remain to be elucidated; however, it may be that the SNP4 G>A mutation results diminished the role of the ISRE in IFN inducibility of the chicken Mx gene, thereby resulting in less expression of the gene, which has been demonstrated to play a role in antiviral function.
Interaction of SNPs can generate the same phenotypic information that can be obtained by individual SNP [24]. Analysis of SNP haplotypes is effective and less expensive. Indeed, in the present study, four haplotypes were generated using SNPs that were in LD (Table 4) and were tested for association with chicken embryos' susceptibility to virulent NDV infection. The haplotype group ACGC was significantly (OR: 9.8, 95\% CI: 1.06-79.43, $P=0.042$ ) associated with chicken embryos' susceptibility to virulent NDV infection. The frequency (0.1) of the haplotype group ACGC was high in the less susceptible group compared to the high susceptible group, which had a frequency of 0.01 . The haplotype group was demonstrated to have the protective effect upon chicken embryo infection with virulent NDV.

Taken together, the polymorphisms of the chicken $\mathrm{Mx}$ gene promoter are associated with chicken embryos' variation in susceptibility to virulent NDV infection. The SNP4 $G>A$ mutation at the $194^{\text {th }}$ position of the amplified region (284 bp) was associated with the phenotype. More importantly, the haplotype group ACGC generated by the combination of SNP1, SNP2, SNP4, and SNP5 was demonstrated to have the resistant effect on chicken embryos infected with virulent NDV. The information is useful for breeding programs designed to develop chicken genotypes that are resistant to Newcastle disease virus. 
TABLE 5: Haplotype analysis of four polymorphic sites of the chicken Mx gene promoter that are in LD and association with chicken embryos' susceptibility to virulent NDV infection.

\begin{tabular}{|c|c|c|c|c|c|c|c|c|c|}
\hline \multirow{2}{*}{ No. } & \multicolumn{4}{|c|}{ Haplotypes } & \multicolumn{3}{|c|}{ Frequencies } & \multirow{2}{*}{ OR $(95 \% \mathrm{CI})$} & \multirow{2}{*}{$P$ value } \\
\hline & SNP1 & SNP2 & SNP4 & SNP5 & Total & $\mathrm{H}$ & $\mathrm{L}$ & & \\
\hline 1 & $A$ & $C$ & $G$ & $T$ & 0.74 & 0.74 & 0.74 & 1.00 & - \\
\hline 2 & $T$ & $G$ & $A$ & C & 0.06 & 0.22 & 0.15 & $0.54(0.21-1.36)$ & 0.2 \\
\hline 3 & $A$ & $C$ & $G$ & C & 0.06 & 0.01 & 0.10 & $9.18(1.06-79.43)$ & 0.042 \\
\hline 4 & $A$ & $G$ & $G$ & $T$ & 0.01 & 0.01 & 0.01 & $0.73(0.04-12.88)$ & 0.83 \\
\hline
\end{tabular}

$\mathrm{CI}$, confidence interval; OR, odds ratio; $\mathrm{H}$, high susceptible chicken embryo group; $\mathrm{L}$, less susceptible chicken embryo group.

\section{Data Availability}

The data used to support the findings of this study are available from the corresponding author upon request.

\section{Conflicts of Interest}

There are no conflicts of interest regarding the publication of this study.

\section{Acknowledgments}

The authors thank the Bill \& Melinda Gates Foundation for funding this research (grant no. OPP1083453) through Program for Enhancing Health and Productivity of Livestock (PEHPL) and the Nelson Mandela African Institution of Science and Technology (NM-AIST) in Arusha, Tanzania, for hosting the program.

\section{References}

[1] O. Haller, H. Arnheiter, J. Pavlovic, and P. Staeheli, "The discovery of the antiviral resistance gene $\mathrm{Mx}$ : a story of great ideas, great failures, and some success," Annual Review of Virology, vol. 5, no. 1, pp. 33-51, 2018.

[2] J. Verhelst, P. Hulpiau, and X. Saelens, "Mx proteins: antiviral gatekeepers that restrain the uninvited," Microbiology and Molecular Biology Reviews, vol. 77, no. 4, pp. 551-566, 2013.

[3] M. B. Uccellini and A. García-Sastre, "ISRE-reporter mouse reveals high basal and induced type I IFN responses in inflammatory monocytes," Cell Reports, vol. 25, no. 10, pp. 2784-2796.e3, 2018.

[4] X. Y. Li, L. J. Qu, Z. C. Hou, J. F. Yao, G. Y. Xu, and N. Yang, "Genomic structure and diversity of the chicken Mx gene," Poultry Science, vol. 86, no. 4, pp. 786-789, 2007.

[5] K. T. Ramasamy, M. R. Reddy, K. S. R. Ravindra, and R. N. Chatterjee, "Chicken Mx gene polymorphisms in Indian native chicken breeds and White Leghorn by real time multiplex allele specific PCR," Indian Journal of Animal Research, vol. 52, pp. 649-651, 2017.

[6] B. Schumacher, D. Bernasconi, U. Schultz, and P. Staeheli, "The chicken Mx promoter contains an ISRE motif and confers interferon inducibility to a reporter gene in chick and monkey cells," Virology, vol. 203, no. 1, pp. 144-148, 1994.

[7] C. G. Yin, L. X. Du, S. G. Li et al., "Expression analysis of the $\mathrm{Mx}$ gene and its genome structure in chickens," Asian-Australasian Journal of Animal Sciences, vol. 23, no. 7, pp. 855862, 2010.

[8] Y. Wang, V. Brahmakshatriya, B. Lupiani et al., "Associations of chicken Mxl polymorphism with antiviral responses in avian influenza virus infected embryos and broilers," Poultry Science, vol. 91, no. 12, pp. 3019-3024, 2012.

[9] J. E. Fulton, J. Arango, R. A. Ali et al., "Genetic variation within the Mx gene of commercially selected chicken lines reveals multiple haplotypes, recombination and a protein under selection pressure," PLoS One, vol. 9, Article ID e108054, , 2014.

[10] J.-H. Ko, H.-K. Jin, A. Asano et al., "Polymorphisms and the differential antiviral activity of the chicken Mx gene," Genome Research, vol. 12, no. 4, pp. 595-601, 2002.

[11] J. H. Ko, A. Takada, T. Mitsuhashi, T. Agui, and T. Watanabe, "Native antiviral specificity of chicken Mx protein depends on amino acid variation at position 631," Animal Genetics, vol. 35, no. 2, pp. 119-122, 2004.

[12] M. A. Pagala, C. Sumantri, and S. Murtini, "Association of Mx gene genotype with antiviral and production traits in tolaki chicken," International Journal of Poultry Science, vol. 12, no. 12, pp. 735-739, 2013.

[13] C. T. O. Benfield, J. W. Lyall, and L. S. Tiley, "The cytoplasmic location of chicken $\mathrm{Mx}$ is not the determining factor for its lack of antiviral activity," PLoS One, vol. 5, Article ID e12151, 2010.

[14] D. J. Alexander and N. J. Chettle, "Procedures for the haemagglutination and the haemagglutination inhibition tests for avian infectious bronchitis virus," Avian Pathology, vol. 6, no. 1, pp. 9-17, 1977.

[15] S. E. Grimes, A Basic Laboratory Manual for the Small-Scale Production and Testing of I-2 Newcastle Disease Vaccine, Food and Agricultural Organization (FAO), Animal Production and Health Commission for Asia and the Pacific (APHCA), Rome, Italy, 2002, http://www.fao.org/3/a-ac802e.pdf.

[16] M. G. Wise, D. L. Suarez, B. S. Seal et al., "Development of a real-time reverse-transcription PCR for detection of Newcastle disease virus RNA in clinical samples," Journal of Clinical Microbiology, vol. 42, no. 1, pp. 329-338, 2004.

[17] L. M. Kim, D. J. King, D. L. Suarez, C. W. Wong, and C. L. Afonso, "Characterization of class I Newcastle disease virus isolates from Hong Kong live bird markets and detection using real-time reverse transcription-PCR," Journal of Clinical Microbiology, vol. 45, no. 4, pp. 1310-1314, 2007.

[18] D. Fleming, J. Koltes, A. Markey et al., "Genomic analysis of Ugandan and Rwandan chicken ecotypes using a $600 \mathrm{k}$ genotyping array," BMC Genomics, vol. 17, no. 1, pp. 407-423, 2016.

[19] R. Osei-Amponsah, B. B. Kayang, and A. Naazie, "Age, genotype and sex effects on growth performance of local chickens kept under improved management in Ghana," Tropical Animal Health and Production, vol. 44, no. 1, pp. 29-34, 2012.

[20] A. Darvasi and M. Soller, "Selective genotyping for determination of linkage between a marker locus and a quantitative trait locus," Theoretical and Applied Genetics, vol. 85, no. 2-3, pp. 353-359, 1992. 
[21] Ś. Sen, F. Johannes, and K. W. Broman, "Selective genotyping and phenotyping strategies in a complex trait context," Genetics, vol. 181, no. 4, pp. 1613-1626, 2009.

[22] C. Mishra, D. Das, P. Kumar et al., "Nucleotide sequencing and PCR-SSCP of Mxl gene in chicken," Indian Journal of Animal Research, vol. 45, pp. 276-282, 2011.

[23] X. Sole, E. Guino, J. Valls, R. Iniesta, and V. Moreno, "SNPStats: a web tool for the analysis of association studies," Bioinformatics, vol. 22, no. 15, pp. 1928-1929, 2006.

[24] E. R. Martin, E. H. Lai, J. R. Gilbert et al., "SNPing away at complex diseases: analysis of single-nucleotide polymorphisms around APOE in Alzheimer disease," The American Journal of Human Genetics, vol. 67, no. 2, pp. 383-394, 2000. 


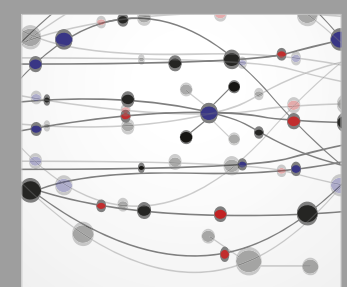

The Scientific World Journal
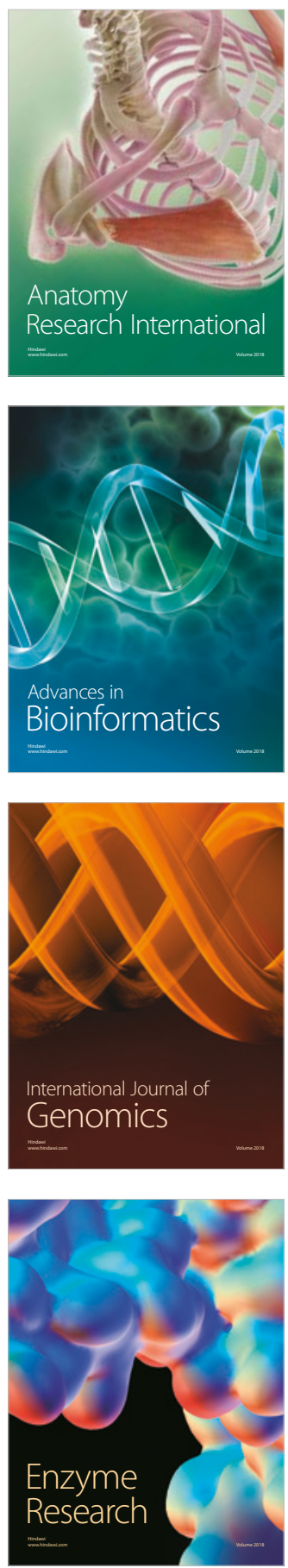
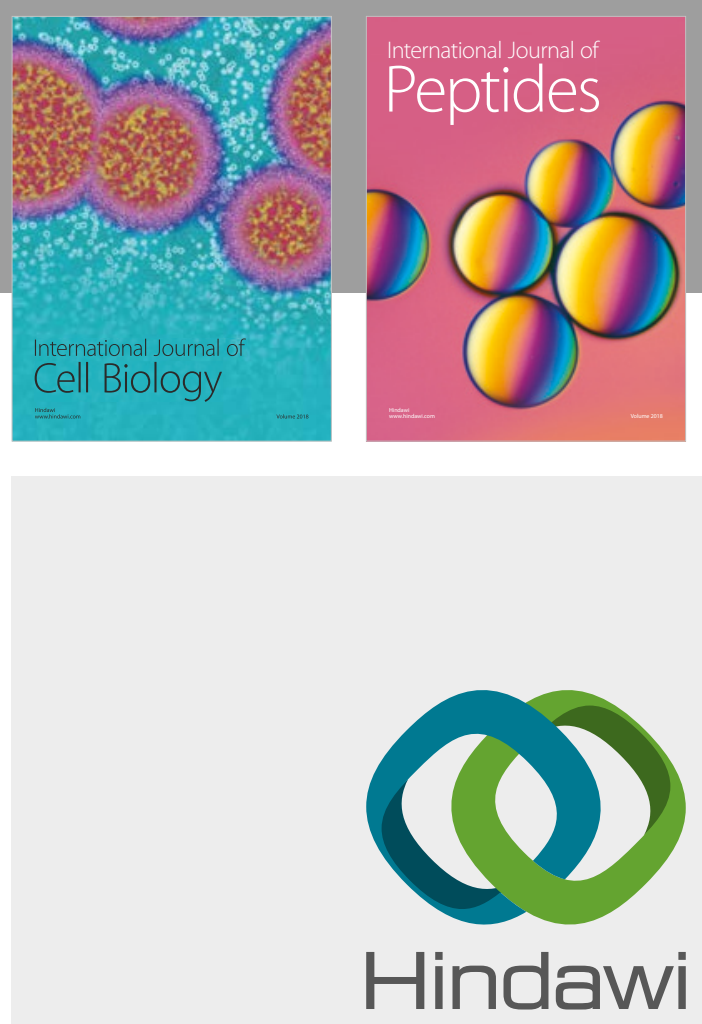

Submit your manuscripts at

www.hindawi.com
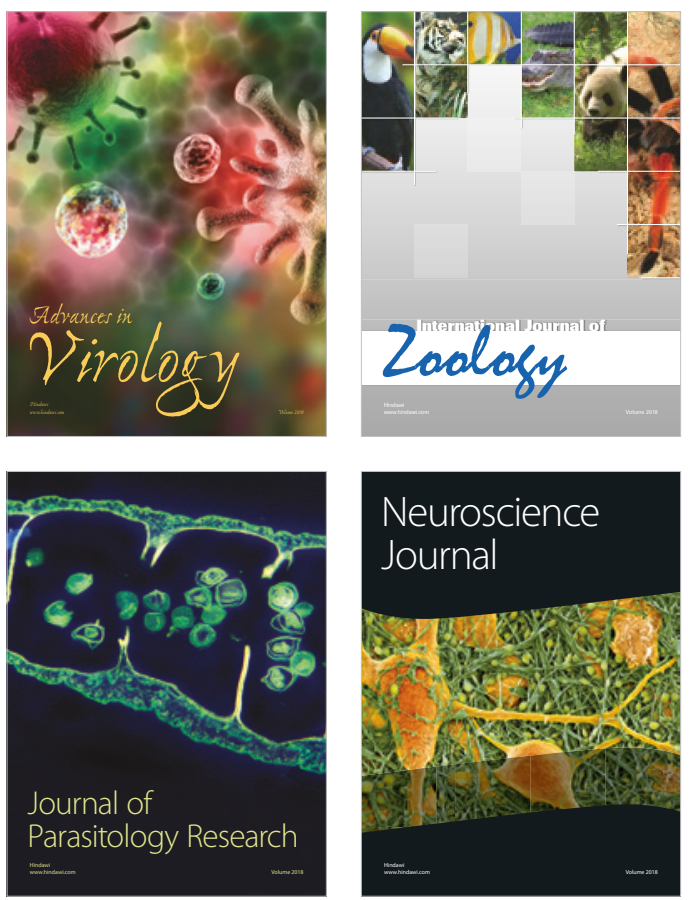
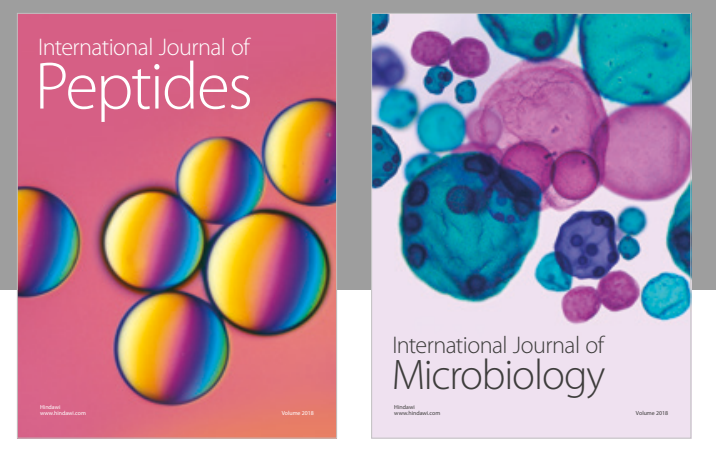

nternational Journal of Microbiology
Journal of
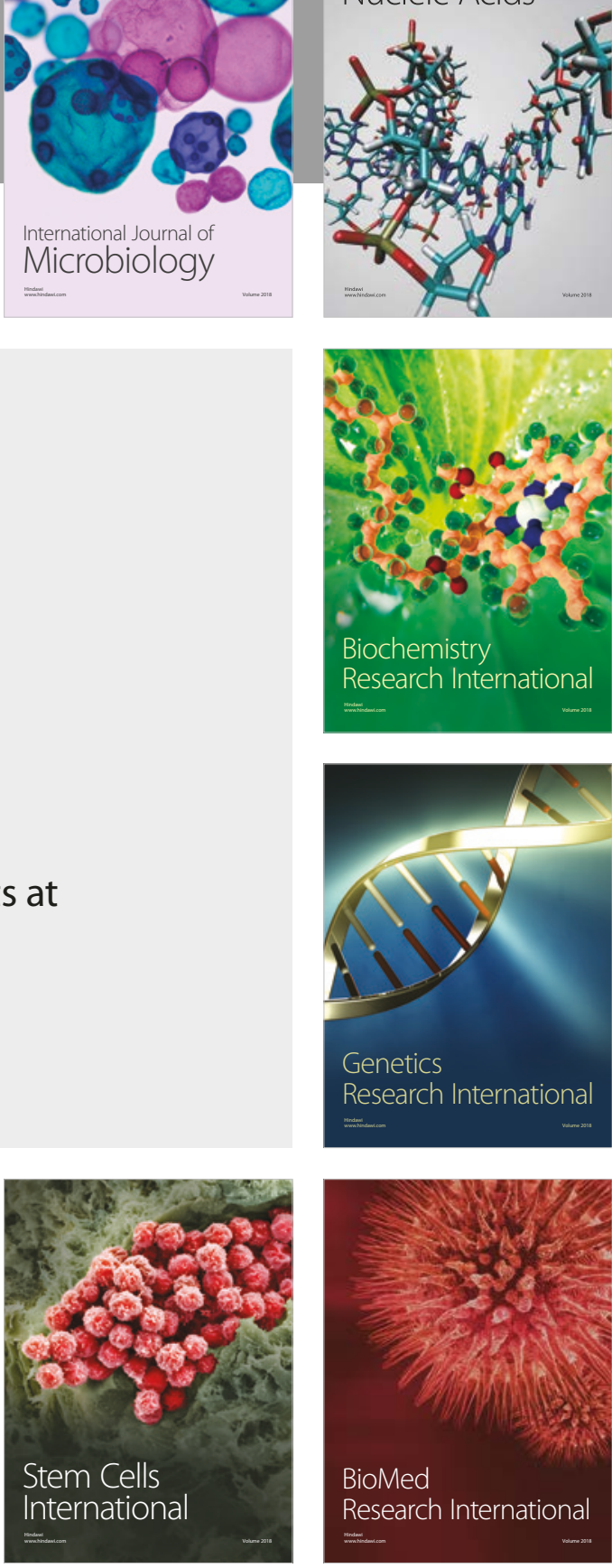
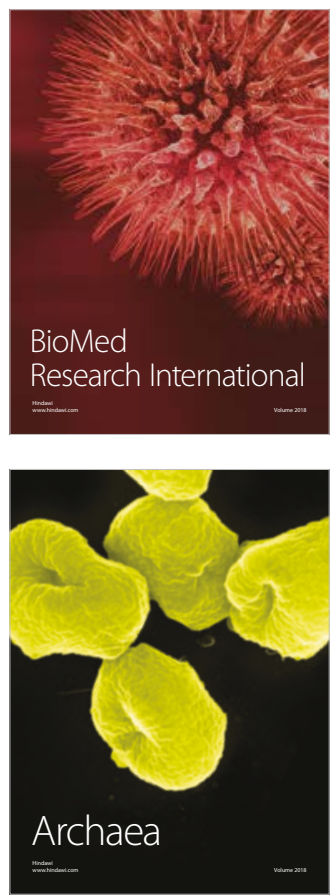